\title{
Interval Point in Time Date and Time Data Type
}

National Cancer Institute

\section{Source}

National Cancer Institute. Interval Point in Time Date and Time Data Type. NCI

Thesaurus. Code C95666.

A data type comprised of a set of consecutive values of an ordered point in time date and time datatype. 